\title{
REPORT
}

\section{Twelve-month-olds point to share attention and interest}

\section{Ulf Liszkowski, ${ }^{1}$ Malinda Carpenter, ${ }^{1}$ Anne Henning, ${ }^{2}$ Tricia Striano $^{2}$ and Michael Tomasello ${ }^{1}$}

1. Department of Developmental and Comparative Psychology, Max Planck Institute for Evolutionary Anthropology, Leipzig, Germany

2. Junior Scientists Group on Cultural Ontogeny, Max Planck Institute for Evolutionary Anthropology, Leipzig, Germany

\begin{abstract}
Infants point for various motives. Classically, one such motive is declarative, to share attention and interest with adults to events. Recently, some researchers have questioned whether infants have this motivation. In the current study, an adult reacted to 12month-olds' pointing in different ways, and infants' responses were observed. Results showed that when the adult shared attention and interest (i.e. alternated gaze and emoted), infants pointed more frequently across trials and tended to prolong each point - presumably to prolong the satisfying interaction. However, when the adult emoted to the infant alone or looked only to the event, infants pointed less across trials and repeated points more within trials - presumably in an attempt to establish joint attention. Results suggest that 12-month-olds point declaratively and understand that others have psychological states that can be directed and shared.
\end{abstract}

\section{Introduction}

Bates, Camaioni and Volterra (1975) first distinguished three types of infant gestures. Ontogenetically earliest are attention-getters in which infants do something to get the adult to attend to themselves: 'showing off'. But most important for subsequent development were two types of triadic gesture involving not only child and adult but in addition an external event or entity. First, protoimperatives involve the infant using the adult as a tool to obtain an object, for instance, pointing as a request for an object. Second, protodeclaratives involve the infant using an object as a tool to obtain adult attention, for instance, showing and pointing as attempts to share attention and interest to an object or event. Bates et al. argued that these triadic gestures were truly intentionally communicative acts, as evidenced by the fact that while gesturing infants often alternated gaze between the adult's face and the object - suggesting that the gesture was intended to affect the adult in some way leading to the desired outcome (see Franco \& Butterworth, 1996, for an elaboration).

Subsequent research has established that protodeclaratives are something special. For example, one of the key diagnostic features of young children with autism is their failure to point for others declaratively - even though they point for others imperatively (e.g. Baron-Cohen, 1991). Relatedly, apes raised by humans typically learn to point for others imperatively, but not declaratively (see Call \& Tomasello, 1996). One interpretation of this difference is that it is one thing to understand other persons as causal agents who make things happen, but it is quite another and perhaps more difficult to understand them as subjects of experience with whom one can share attention and interest (Tomasello \& Camaioni, 1997). However, research in which the developmental emergence of these two types of pointing gesture are systematically compared in typically developing children finds that they emerge together at around 12 months of age, with declaratives emerging a bit earlier than imperatives (Carpenter, Nagell \& Tomasello, 1998; Bretherton, McNew \& Beeghly-Smith, 1981; Leung \& Rheingold, 1981). So apparently typically developing children do not find declaratives more difficult than imperatives.

Another explanation for this difference is that typically developing children have the motivation to share attention and interest with others to external objects and events but children with autism and apes do not have this motivation (e.g. Tomasello, 1999). However, motivation is not easily observed. Sometimes the declarative 
motive is inferred based simply on the lack of an imperative motive: if the infant does not desire the object she must be attempting to share attention to it (e.g. SavageRumbaugh \& Fields, 2000). But this is not sufficient because, as proposed by, for example, Werner and Kaplan (1963), infants sometimes point to things nonsocially - just to orient their own attention (see also Blake, O'Rourke \& Borzellino, 1994). Also, pointing may sometimes be used as a kind of recognitory gesture best glossed as 'I know that thing' (Piaget, 1962). And it is at least theoretically possible that an infant might point communicatively only wanting the adult to look at something - with no motive to share attention. The main point is that the declarative motive cannot simply be inferred from the absence of an imperative motive; sharing attention is a distinct motivation that must be positively distinguished from other motivations such as self-directing, recognition and simply wanting the adult to look.

Moore and Corkum (1994) have expressed skepticism that 12-month-olds produce declarative gestures. They contended that early points typically identified as protodeclaratives are in reality aimed at gaining adult attention to the self. Moore and D'Entremont (2001) addressed this question empirically, arguing for a learning account on which protodeclarative points do not emerge until 18 months of age. They claimed that what young infants are really doing is attempting to get a self-related emotional reaction from the adult. Their main evidence for this interpretation is that 12 -month-olds seldom attempt to redirect the adult's attention to new things, quite often pointing to things the adult is already looking at. Underlying this line of reasoning is the assumption that to point declaratively the infant must desire to share attention to something the adult is not already focused on; that is, the claim is in effect that a part of the declarative motive is a kind of informing motive (see also Franco \& Gagliano, 2001). But although this informing motive is interesting itself from a social-cognitive point of view, it is not always a necessary condition for sharing attention and interest. Two people may already both be looking at something, but one may nevertheless want to express her interest in it and so convince the other to share this attention and interest. Children thus point declaratively, in our view, both to direct attention (even if the adult is already looking), and to elicit a reaction from the adult indicative of shared attention and interest, that is, a comment of some sort. Both goals are necessary for declarative pointing and only together are they sufficient. In other words, the infant wants the adult to (i) attend to the event and (ii) give a comment back. The latter is not possible without the former and the former is not enough for sharing attention and interest.
In the current study we attempted to test infants' motives for pointing directly, to contrast different theories of what infants want when they initially begin pointing. We elicited points in a declarative context with objects either suddenly appearing or engaging in interesting actions from afar (out of reach; see Butterworth \& Grover, 1990) or else abstract or non-graspable stimuli (see Perucchini, 1997). We then experimentally manipulated the adult's reaction and observed the infants' responses to this reaction. More specifically, we tested four hypotheses:

- Infants want nothing from the adult; they are pointing for themselves

- Infants want adult attention and emotion to themselves

- Infants want to direct the adult's attention to the object

- Infants want to share attention and interest to the object

We measured in infants' behavior signs of satisfaction and dissatisfaction with the adult's reaction: the number of events at which infants pointed across the entire session, whether they pointed a second or third time for the same referent within an event, the duration of the point(s), and whether infants looked to the adult's face.

Based on the theoretical proposals of Tomasello (1999; see also Carpenter et al., 1998), we expected that infants would behave in a distinctive manner when the adult shared attention and interest as compared with the other experimental conditions - since infants really do have a declarative motive at 12 months of age. Although it was not clear at the outset precisely what infant behaviors to expect in each of the conditions, we expected infants to be dissatisfied with the adult's response in the three conditions in which she did not share attention and interest. One possibility is that infants would repeat their pointing more often when the adult looked only to the object or only emoted positively toward them - since these behaviors were not the desired reaction of sharing attention and interest (i.e. behavioral persistence when one's goal is not met; see Shwe \& Markman, 1997, and Franco \& Gagliano, 2001, for examples of this with older children). When the adult shared attention and interest with infants, we expected that they would either be satisfied with this reaction and cease pointing relatively quickly or that they would attempt to prolong the pleasing interaction of sharing attention by prolonging their point for as long as the adult was engaged in the desired attention-sharing behaviors.

\section{Pilot study}

In an initial pilot study, we elicited pointing in a declarative context from 23 12-month-old German infants. We 
used a within-subjects design, counterbalanced for order across children. During test trials infants sat on the floor with their mothers behind them and were shown hand puppets appearing through a door for 10 seconds. Immediately after each infant point, an adult experimenter (E1) reacted for 10 seconds in one of four different ways ( 2 consecutive blocks of all four reactions, totalling 8 trials):

Joint Attention: E1 repeatedly looked back and forth between the event and the infant's face, talking excitedly about the stimulus and commenting on the fact that they were seeing it together. For example, she said, 'Oh, wow! What's that? Are you showing Grover to me? Yes, he is blue.' Face: E1 looked at the infant's face, never looking at the event, and talked excitedly but only about the infant. For example, she said, 'Oh, I see you are in a good mood! Did you sleep well? Oh, your eyes are big!'

Event: E1 looked only at the event, never looking back to the infant, and did not speak or show excited emotions. Ignore: E1 looked only at her hands, never looking at either the infant's face or the event. Instead, she picked at her nails, rubbed her fingers, or brushed her hands on her leg in a natural way, showing no excited emotions.

The main difference found was that infants prolonged their points longest in the joint attention condition: main effect of point duration, $F(3,66)=5.52, p=.002$, with simple effects for Joint Attention $(M=3.07$ seconds) compared to the Event $(M=2.22$ seconds, $p=$ $.025)$ and the Ignore $(M=1.35$ seconds, $p<.001)$ conditions. Although infants pointed almost $1 \mathrm{sec}$ longer in the Joint Attention than in the Face condition $(M=2.16$ seconds) this difference was not statistically significant $(p=.145)$. No other measures produced statistically significant differences.

These results are fairly weak. The main problem, we believe, was the within-subjects design, which made it difficult to assess infants' preference for conditions (because conditions changed after each trial). Further, not all infants pointed in each condition (since there were only two trials per condition) leaving many empty cells. There were also indications of order effects - children becoming bored or fatigued over time and not as a function of condition. We therefore changed to a betweensubjects design.

\section{Main study}

In the main study we improved the pilot study's procedure in several ways. Most importantly, we increased the number of infants and had each infant participate in only one experimental condition. This enabled us to give more trials to infants within conditions and thus to look at changes in pointing across trials as a function of the social partner's interaction. We made several other smaller changes as well. First, we did not constrain the stimulus exposure to $10 \mathrm{sec}$ to give infants more time to initiate a point. Second, we altered the seating arrangement so that mothers were less involved (in the pilot study infants occasionally pointed for their mothers). Finally, we used a set of different stimuli and displayed them through 'windows' at different locations to make the underlying motive for pointing even more likely to be declarative - because the stimuli were more clearly unobtainable (in some cases even non-physical, i.e. colored lights).

\section{Method}

\section{Participants}

German infants were recruited from a database of infants whose parents had agreed to participate in studies of infant development. The majority were firstborns and with a middle-class background. When recruited, parents were asked whether their infant pointed already. In all, 112 infants who were reported to point were tested. Infants were excluded because of experimenter error (3) or fussiness (8), or because they did not point during the test session (26). The final sample thus included 75 infants (44 girls, 31 boys; mean age: 12:6; range: 11:16 to 13:20). Infants were randomly assigned to one of the four experimental conditions with gender being balanced across conditions ( $n \mathrm{~s}$ for Joint Attention $=18$, Face $=18$, Event $=19$, Ignore $=20$ ). Infants received a small gift for participating.

\section{Materials}

Testing took place in a $4.5 \times 3.5 \mathrm{~m}$ testing room (see Figures 1 and 2). The infant was seated in a child-sized chair (about $50 \mathrm{~cm}$ high) with a small table attached to it. Attached to the table was a toy $(20 \times 20 \mathrm{~cm})$ with large, moveable beads which had been shown to be neither too attractive nor too boring for the infant during pilot testing. E1 sat at 90 degrees to the infant's right and the mother sat at 90 degrees to the infant's left. E2 hid behind a large screen made of white cloth sheets which blocked the entire back side of the testing room. The screen was positioned in front of the infant at a distance of $2.5 \mathrm{~m}$. It measured $4.5 \times 2.1 \mathrm{~m}$ and had six evenly spaced window openings $(0.5 \times 0.5 \mathrm{~m}, 15 \mathrm{~cm}$ apart, $90 \mathrm{~cm}$ from the floor). These openings were positioned at about 13, 27 and 37 degrees left and right from the infant's midline and were covered with curtains 


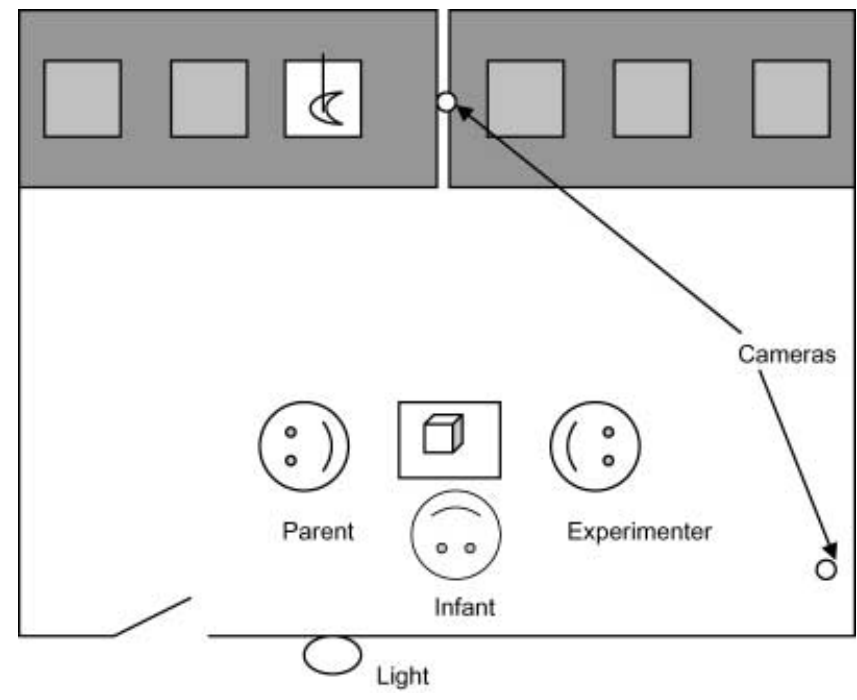

Figure 1 The testing set-up.

which could be raised and lowered with a string from behind. Behind each opening a different object hung from the upper frame of the screen, neither touching the screen nor being visible when the curtains were closed. During test trials, E2 operated the objects from behind the screen.
A total of 10 stimuli were used for the different test events. None of the events produced sound. Six were toys that moved in some way: two different wooden birds whose wings flapped, two different stuffed animals who danced in the air on strings, and two different hand puppets who moved animately. Four of the stimuli were lights: there were two differently colored strings of lights (yellow and white) which turned on and off at 1 -sec intervals, and two moving light patterns produced by spotlights. However, because the spotlights rarely elicited any points, they were replaced by two further hand puppets for the second half of participants. The windows close to midline were re-used for two (stimuli-set with spotlights) or three (stimuli-set without spotlights) stimuli. The positions of the toys were fixed.

One camera, positioned at the infant's midline and hidden behind the screen, recorded the infant, E1 and mother. A second camera, also hidden from the infant, was positioned in a corner opposite to the screen, and recorded the order and timing of event exposure. Both cameras were connected to a quad-splitter that fed the image into a monitor behind the screen so E2 could monitor the infant's behavior. A light behind the infant was switched on by E2 to signal to E1 the onset and offset of each stimulus.

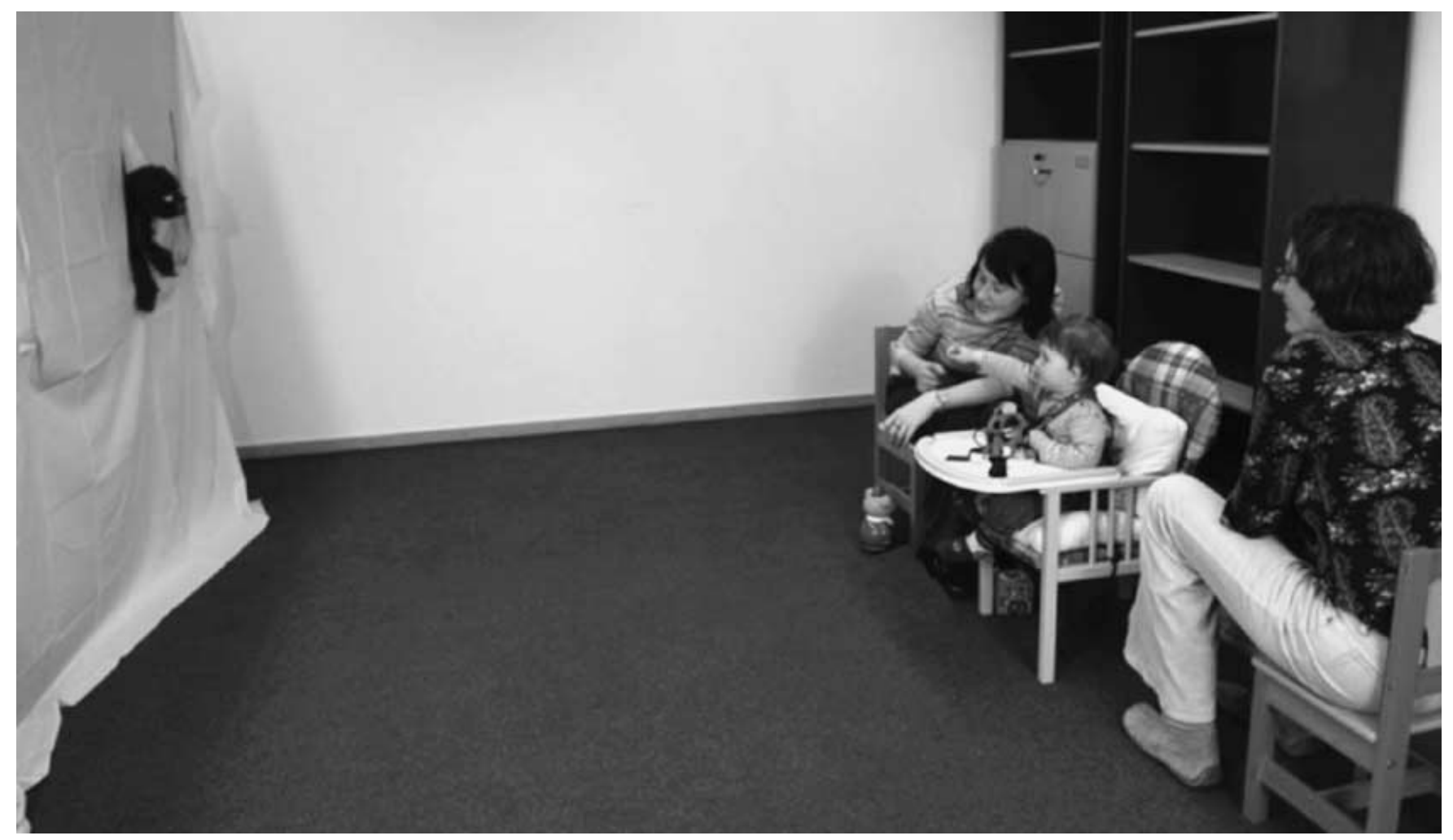

Figure 2 Staged photograph of a testing situation. The infant points to Grover and E1 reacts with Joint Attention. 


\section{Procedure}

During warm-up play between E1 and infants, mothers were instructed not to initiate any communicative behavior toward infants during testing and not to look at the screen at any time. The session began with a brief play period with the bead toy to keep the infant interested in E1 as a social partner (this type of play was also repeated between test trials). When E1 judged that the infant was relaxed and attentive, she gradually (but naturally) withdrew from interaction: she stopped touching the toy, addressing the infant verbally, and establishing eye contact. She then signalled E2 to start the trial by touching her ear. E2 switched on a light which signalled to E1 the stimulus onset, opened a curtain, and made the assigned object move. E1 ignored the stimulus until the infant pointed, whereupon she reacted continuously for the duration of $10 \mathrm{sec}$ according to one of four conditions: Joint Attention, Face, Event, Ignore (see pilot study for description). E1 attempted to produce the same intensity of emotions in the Joint Attention and Face conditions ${ }^{1}$ and controlled for the amount of verbal utterances by an internalized rhythmic structure. After the $10 \mathrm{sec}$ had elapsed, the curtain was closed and E1 returned to normal play interaction with the infant. There was no set time limit for stimulus exposure before the point, but if the infant did not point and looked away for more than 2 seconds, the stimulus was removed and the curtain closed. There was a total of 10 trials. If infants pointed outside of the test event (e.g. to a different, closed window) E1 did not follow their point and briefly commented on the behavior (e.g. 'Hmm, that was a nice point'). She then drew the infant's attention back to the bead toy.

\section{Coding and analyses}

We were primarily interested in infants' points and looks to E1 during the $10-\mathrm{sec}$ test trials. Points were coded when the infant extended the arm (either fully or slightly bent) and index finger or open hand, palm down, in the direction of the stimulus. Point duration was coded from the onset of the movement which resulted in a point until the arm was drawn back and the index finger or hand was no longer extended (e.g. Murphy \& Messer, 1977). Points to non-test objects were not coded. Looks to E1 were coded only when infants looked to E1's face.

\footnotetext{
${ }^{1}$ A coder blind to the hypotheses rated the emotionality of E1's reactions on a scale from 0 to $5(0=$ no emotion; $3=$ average emotion; $5=$ strong emotion) for $25 \%$ of infants in these two conditions. El's emotionality was rated between 2 and 4 with a median of 3 and no statistical differences between conditions, $\chi^{2}(2,52)=4.58, p=.101$.
}

Coding was done in different real-time passes by a research assistant who was blind to the hypotheses of the study with a specialized program (Interact, Version 6.5; Thiel, 1991) with on/off activation of a keyboard key corresponding to the occurrence and duration of the relevant behaviors. Inter-observer reliability was assessed by E2 who coded $20 \%$ of the infants' tapes. Reliability was excellent: Cohen's Kappas on a 1-sec time-base were .90 for duration of points, .90 for number of points, .97 for looks to E1 and .96 for latency of point onset.

There were six main measures:

Number of trials with a point: How many times E1's response was triggered during the testing session (i.e. the mean proportion of trials for which the infant pointed at least once).

Number of points within a trial: Mean number of points during a trial.

Duration of points: Mean duration of points during a trial. Latency to point: Mean time interval between stimulus onset and point onset.

Number of looks to E1 during or after the point: Mean number of self-initiated looks (i.e. not in response to something E1 said) to E1's face per trial.

Number of looks to E1 before the point: Mean number of looks to E1's face after the stimulus onset and before the point.

We conducted one-way ANOVAs with condition as a between-subjects factor and post-hoc tests (LSD, two-tailed) on these six dependent measures. Nine infants pointed only once (three in each of the Face, Event and Ignore conditions). Since all dependent measures consisted of mean proportions relative to points except for the measure number of trials with a point (which was based on proportions relative to the number of trials), the nine infants who produced only one point were not included in overall analyses on those measures (those $n$ s thus $=66$ ).

\section{Results}

Preliminary analyses confirmed that there was no effect of gender on any measure, so the variable was ignored in further analyses.

\section{Proportion of trials with a point}

Since a few infants (6) were shown less than 10 stimuli (because of fussiness or experimenter error) this analysis was carried out on mean proportions. A one-way ANOVA yielded a significant effect of condition, $F(3,74)=4.30$, $p=.008$. Post-hoc tests showed that infants in the Joint Attention condition had the highest number of trials with a point as compared with each of the other three conditions (all $p \mathrm{~s}<.031$ ). No other differences between 


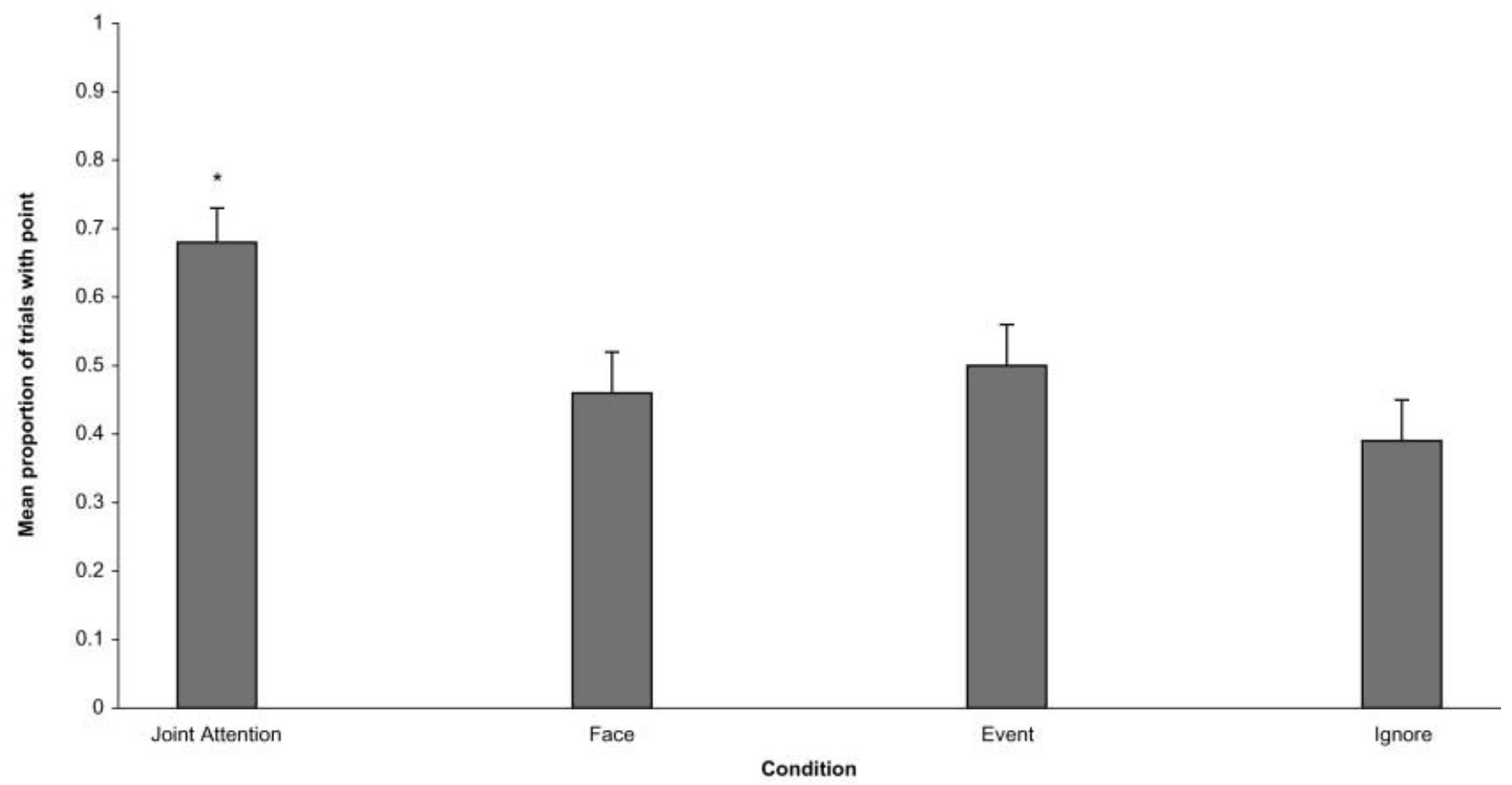

Figure 3 Number of trials with a point for each condition. * indicates a significant difference $(p<.05)$ compared to all other conditions.

conditions were significant (see Figure 3). Thus, sharing interest in the novel event was the adult reaction that seemed most likely to increase infants' motivation to point when the new stimuli appeared in the succeeding trials.

\section{Number of points per trial}

A one-way ANOVA on mean number of points per trial yielded a significant effect of condition, $F(3,65)=3.03$, $p=.036$. Post-hoc tests showed that infants in the Joint Attention condition pointed less often than infants in the Face, Event and Ignore conditions $(p s=.013, .013$ and .05 , respectively). There were no significant differences between the latter three conditions (see Figure 4). Thus, infants significantly increased their frequency of pointing within trials if the adult did not look at and comment back on the event as in the Joint Attention condition. ${ }^{2}$

\footnotetext{
${ }^{2}$ We also looked for differences between conditions in the first trial to see whether infants responded immediately to El's reaction differentially. A one-way ANOVA on the mean number of points in the first trial revealed no significant differences across conditions although the pattern of results was similar to the previous analysis (Joint Attention $M=1.05$; Face $M=1.17$; Event $M=1.20$; Ignore $M=1.37$ ), mainly because only 12 out of 75 infants pointed more than once on the first trial. The distribution of subjects who produced more than one point versus only one point in the first trial showed a similar pattern as the analysis of means: Joint Attention: 1/17; Face: 2/16; Event: 4/16; Ignore: 5/14 $\left(\chi^{2}=n s\right)$.
}

\section{Duration of points}

A one-way ANOVA on mean duration of points revealed a marginally significant effect of condition, $F(3$, $65)=2.67, p=.055$. Post-hoc tests showed that the mean duration of points per trial was longer in the Joint Attention condition than in the Event $(p=.01)$ or Ignore $(p=$ .037) conditions (see Figure 5). Although infants' points in the Joint Attention condition were on average almost a full second longer than in the Face condition, this difference was not statistically significant $(p=.130)$. The Face condition was also not significantly different from either of the other two conditions. ${ }^{3}$ Thus, infants kept up their point reliably longer in the Joint Attention condition than in the Event or Ignore conditions.

\footnotetext{
${ }^{3}$ Again we looked for differences between conditions in the first trial. A one-way ANOVA on the mean duration of points in the first trial revealed a similar pattern of results. There was a significant effect of condition, $F(3,71)=2.79, p=.047$, and post-hoc tests showed that mean duration of points was longer in Joint Attention $(M=4.2)$ than Event $(M=2.20 ; p=.009)$ or Ignore $(M=2.47 ; p=.024)$. Infants' points were more than one second longer in the Joint Attention than in the Face condition $(M=3.03)$, but this difference was not statistically significant $(p=.126)$. The Face condition was also not significantly different from either of the other two conditions.
} 


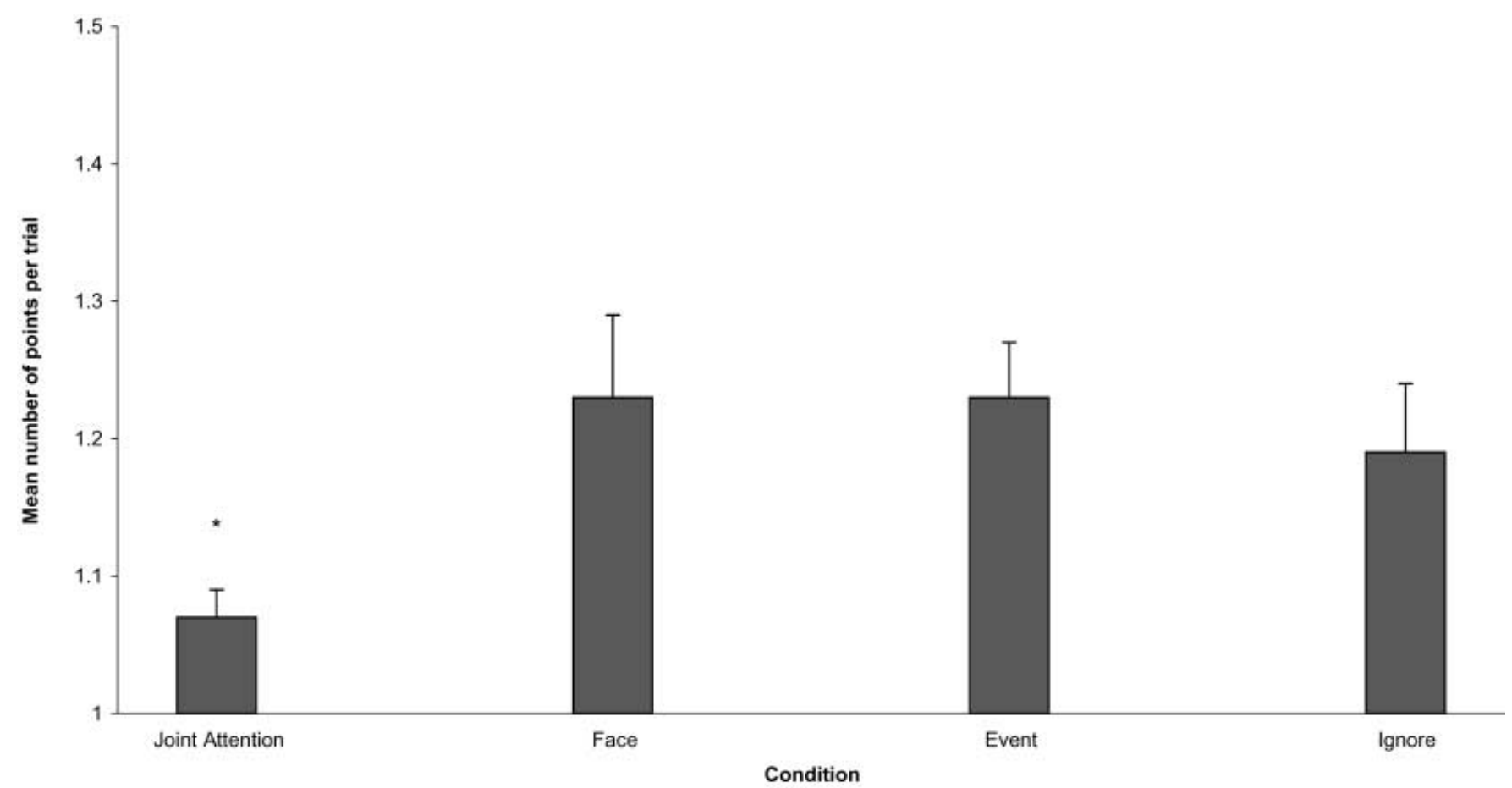

Figure 4 Mean number of points per trial for each condition. * indicates a significant difference $(p<.05)$ compared to all other conditions.

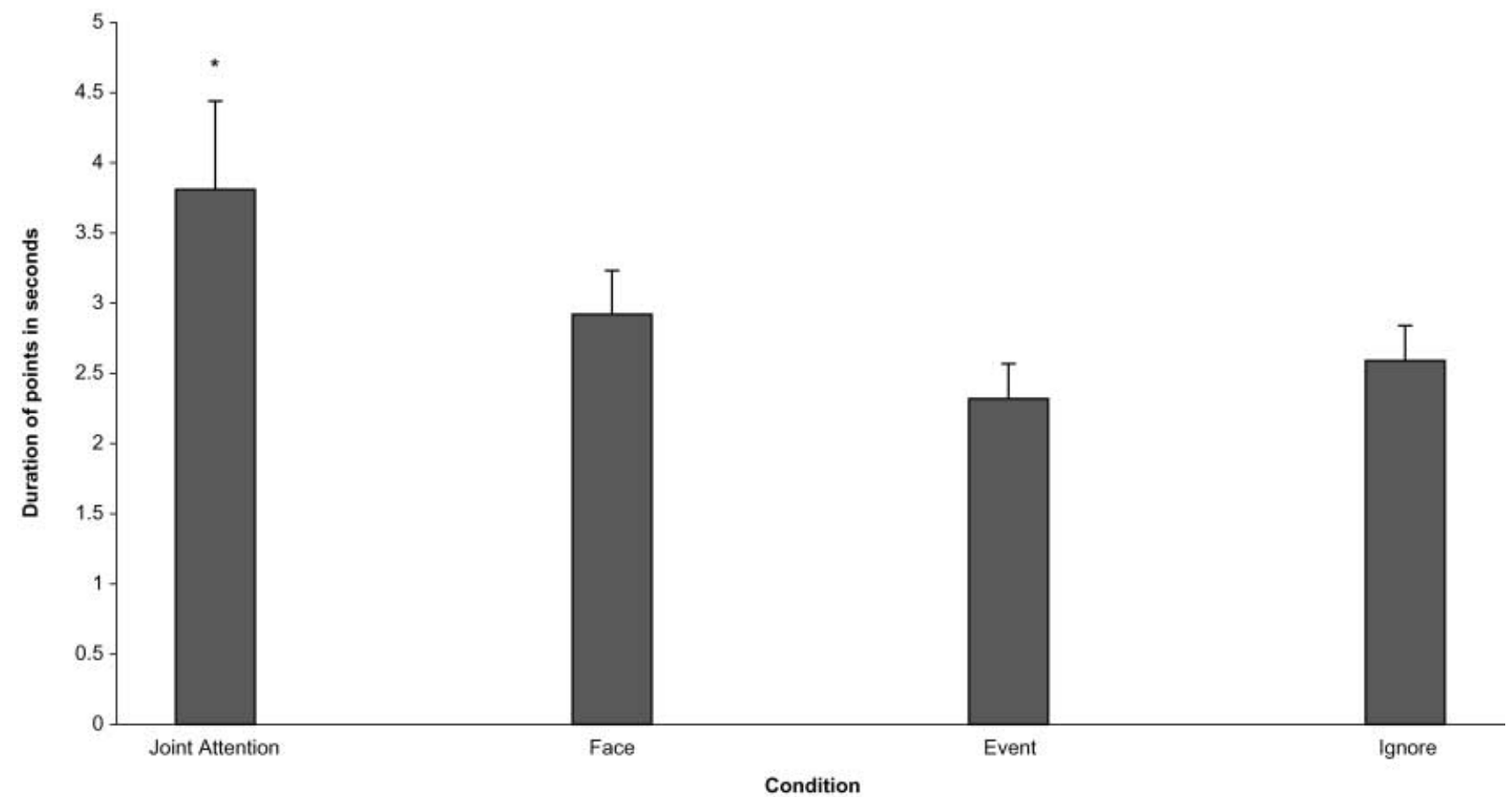

Figure 5 Mean duration of points for each condition. * indicates a significant difference $(p<.05)$ compared to Event and Ignore conditions.

(C) Blackwell Publishing Ltd. 2004 


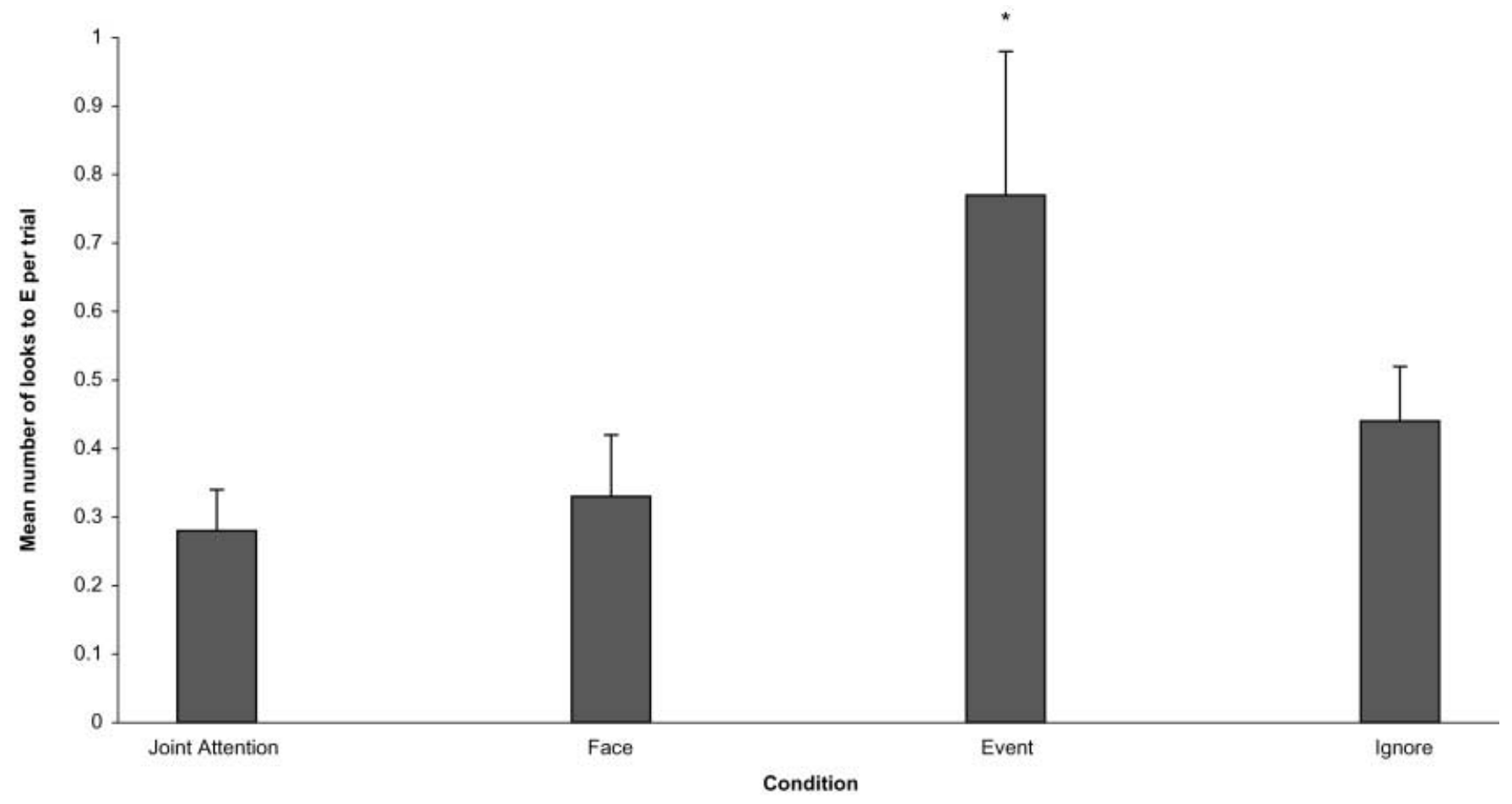

Figure 6 Mean number of looks to E1's face during or after pointing for each condition. * indicates a significant difference ( $p<$.05) compared to all other conditions.

\section{Latency to point}

Although children were on average several seconds quicker to point in the Joint Attention condition as compared with the others (Joint Attention $M=9.76$; Face $M=$ 15.28; Event $M=12.91$; Ignore $M=12.67$ ), a one-way ANOVA on mean latency to point revealed no significant differences across conditions.

\section{Number of looks to E1}

A one-way ANOVA on the mean number of looks to E1 during or after pointing revealed a significant effect of condition, $F(3,65)=4.96, p=.004$. Post-hoc tests showed that infants looked to E1 significantly more often in the Event than in the Joint Attention, Face and Ignore conditions ( $p s=.003, .001$ and .031 , respectively; see Figure 6). Infants thus alternated their gaze between the event and the adult most often when the adult was only attending to the event and not commenting on it. ${ }^{4}$

\footnotetext{
${ }^{4}$ To control for the possibility that many self-initiated looks in the Joint Attention and Face condition had been excluded because E1 directly addressed the infant, we conducted the same analysis on all looks to E1 regardless of whether they were self-initiated or not. A one-way ANOVA yielded a significant effect of condition, $F(3,65)=3.18$,
}

A one-way ANOVA on the mean number of looks to E1 that occurred before pointing did not reveal significant differences across conditions. Overall, infants did not look to E1 very often before initiating a point: Joint Attention $M=0.22$; Face $M=0.22$; Event $M=0.36$; Ignore $M=0.19$.

\section{Summary of results}

Table 1 presents a summary of the main results of the statistical analyses (i.e. columns correspond to Figures 3-6). Results show statistical differences between conditions for each measure.

\section{Discussion}

The motive to share attention and interest with others is a uniquely human motivation and it would seem to be a purely social motive. Much of human culture is predicated on individuals having this declarative motivation,

$p=.030$, and post-hoc tests again showed that infants looked to E1 significantly more often in the Event than in the Joint Attention or Face conditions ( $p \mathrm{~s}=.006, .018$, respectively). The difference between the Event $(M=.77)$ and Ignore $(M=.44)$ conditions was marginally significant $(p=.063)$. 
Table 1 Summary of main statistical analyses (Figures 3-6)

\begin{tabular}{lcccc}
\hline & $\begin{array}{c}\text { Number of } \\
\text { trials with } \\
\text { point (Fig. 3) }\end{array}$ & $\begin{array}{c}\text { Number of } \\
\text { points per } \\
\text { trial (Fig. 4) }\end{array}$ & $\begin{array}{c}\text { Duration } \\
\text { of points } \\
\text { (Fig. 5) }\end{array}$ & $\begin{array}{c}\text { Looks } \\
\text { to E1 } \\
\text { (Fig. 6) }\end{array}$ \\
\hline Joint Attention & + & - & + & - \\
Face & - & + & 0 & - \\
Event & - & + & - & + \\
Ignore & - & + & - & - \\
\hline
\end{tabular}

Note: (+) indicates values statistically higher than (-) in the column; (0) indicates a figure not statistically different from any of the others in the column.

and its absence in children with autism is both a source and a symptom of many of their problems.

In the current study we demonstrated that when infants are first beginning to point around the age of 12 months they point for others with a declarative motive. When interacting with an adult who always reacted consistently in one of four ways, 12-month-olds pointed most often across trials if the adult actively shared her attention and interest in the event (Joint Attention condition). If, on the other hand, the adult only looked to the event while ignoring the child (Event condition), or only looked and emoted to the child while ignoring the event (Face condition), or ignored both (Ignore condition), infants pointed less often across trials. This difference between conditions excludes the possibility that the infants were totally stimulus-driven and pointed simply for themselves; the social context within which the pointing took place - specifically, the partner's reaction - was crucial.

Analyses of infants' points within each event revealed a complementary set of results. In the conditions not involving joint attention, infants repeated their point more often. This repeating behavior presumably indicates that they were dissatisfied with the adult's response, and so they were persisting in their pointing behavior hoping eventually to obtain the desired response (which was presumably joint attention, since children did not repeat themselves very often in this condition). A variety of studies show that older children respond in exactly the same way in similar circumstances using language: when the adult does not respond as desired they repeat or repeat with modification what they have just said (Shwe \& Markman, 1997; Tomasello, Farrar \& Dines, 1984).

Interestingly, infants' individual points lasted longer in the Joint Attention condition than in either the Ignore or Event conditions; the Face condition was in between, differing from none of the others. Given that the Joint Attention and Face conditions, but not the other two, involved the adult displaying positive emotions to the child, prolonging pointing may be a way of maintaining this interaction. This might provide some limited support for the hypothesis of Moore and D'Entremont (2001) that infants point merely to get an adult emotional reaction to them (or else, that infants were distracted by E1's emotional expression and thus forgot to lower their arms). However, the other results (more pointing across trials in the Joint Attention condition but more repeating of points in the Face condition) demonstrate clearly that obtaining an adult's emotional reaction to the self cannot be the whole story. Infants desire an adult's positive emotional reaction to them - and so they were pleased with the Face response even if it was not what they wanted initially - but in the current context this alone was not sufficient.

And finally, it is interesting that infants looked more to the adult's face in the Event condition than in any of the others. One interpretation of this behavior is that infants were puzzled that the adult was looking at the designated event but not showing a reaction about it to them.

Our overall interpretation of infants' behavior in the four experimental conditions, following the pattern of results displayed in Table 1, is thus as follows.

- Ignore condition: infants were not satisfied with the adult's response.

- Event condition: infants were not satisfied with the adult's response, and they were also a bit puzzled by it (so they looked at the adult's face).

- Face condition: infants were not satisfied with the adult's response, but they liked the emotional reaction anyway (so they prolonged their point a bit).

- Joint Attention condition: infants were satisfied with the adult's response.

We thus believe that when infants first begin pointing, they do so (in this situation) to share attention and interest with other people. Other explanations of the results involving learning without any understanding of psychological states cannot easily account for the distinctive pattern of results we found. For example, in Moore and colleagues' account (Moore \& Corkum, 1994; Moore \& D'Entremont, 2001), infants would be satisfied if the adult reacted towards them, but indifferent to whether the adult was integrating the event into the interaction ('the infant is initially not seeking to produce some object-oriented reaction from the adult partner'; Moore \& D'Entremont, 2001, p. 127). Thus, on such an account one might expect that infants would be less satisfied in our Joint Attention condition as compared with the Face condition because they were receiving less attention to the self. However, our overall pattern of results contradicts this interpretation (see Table 1). In general, it is difficult to explain by any learning account precisely why infants should point longer and to more different events across trials in the Joint Attention condition, but then point more often within an event in the other conditions 
without invoking the hypothesis that children are trying to influence the partner's attention and interest. Instead, we think that our overall pattern of results is consistent with other studies of infant pointing and early social cognition suggesting that infants are indeed sensitive to the interest and attention of other persons, and their declarative pointing reflects this (e.g. Baron-Cohen, 1991; Bates et al., 1975; Blake, 2000; Bretherton et al., 1981; Butterworth, 1991; Butterworth, Franco, McKennzie, Graupner \& Todd, 2002; Carpenter et al., 1998; Franco \& Butterworth, 1996; Henderson, Yoder, Yale \& McDuffie, 2002; Leung \& Rheingold, 1981; Perucchini, 1997). It is also important that from about 13 or 14 months of age many infants will be making active attempts to manipulate the interest and attention of others with linguistic symbols - and there is no good reason to suspect that this operates on totally different principles than pointing (Tomasello, 1999).

In our view, the declarative motive has two components. The first is that the partner should attend to the same thing as me, and the second is that she should 'comment' on some aspect of this back to me. The first component requires that infants are sensitive to whether another person is or is not focused on their object of attention and that they understand that another person's attentional state can be directed with a communicative gesture. This is in line with Moore and D'Entremont's (2001) findings, as their infants did sometimes point to an event when the adult was not looking at it. But since these infants also pointed to the event when the adult was already looking at it, Moore and D'Entremont (2001) concluded that 12-month-olds did not point to direct attention and that infants thus do not 'understand the independent attentional perspective of the interactive partner' (p. 128). In light of the current study, we interpret their findings differently. We think that: (i) infants did point to direct the adult's attention to an interesting event, and (ii) when the adult was already looking at the event, infants pointed to elicit a comment about the event they were jointly attending to, i.e. they wanted to share attention and interest.

In other words, at the basis of all forms of pointing is a motive to direct another person's attention. Evidence that 12-month-olds do indeed understand that others attend to only some things within their visual field comes from a study by Tomasello and Haberl (in press), who found that by around their first birthdays infants understand something specific about other persons' attention based on what the two of them have or have not experienced together. The declarative motive then adds in the desire for a comment back from the person whose attention has been directed (whereas other types of pointing add in other second components). It is possible that this desire originates from earlier dyadic interactions in which adults share emotions and engage in contingent exchanges with the infant (e.g. Gusella, Muir \& Tronick, 1988; Muir \& Hains, 1993; Rochat \& Striano, 1999). Nevertheless, it is not clear from the current study exactly what needs to be in the comment. One can imagine that the infant wants the adult to express excitement about exactly that aspect of the event she is focused on (our response in the current study was fairly generic), or that she is trying to gather information from the adult's comment on the event (see Baldwin \& Moses, 1996). It is also possible that infants would be satisfied with some types of emotional reaction but not others; for example, it is not clear if they would be satisfied if the adult expressed a negative emotion to them about the shared event or simply acknowledged receipt of information without expressing emotions or signs of really sharing attention and interest (in the current study there was always positive emotion expressed in the Joint Attention condition and the adult never commented back in the Event condition). In any case, future research should attempt to address these different possibilities for infants' expectations about the comment component of the adult's reaction to their declarative pointing.

Taken together, this study suggests that infants at 12 months of age do understand something about attention and the independent attentional perspective of others when sharing attention and interest - a suggestion that is consistent with recent findings using other paradigms (Moses, Baldwin, Rosicky \& Tidball, 2001; Tomasello \& Haberl, in press). With this understanding, infants actively engage in mutual informational exchanges about events in the outside world with the goal of sharing attention and interest.

\section{Acknowledgements}

The study is based on discussions with Chris Moore. We thank Josep Call for his help with the design of this study. We are grateful to the Universitätsfrauenklinik for support and assistance with subject recruitment and to the parents and infants for participating in the research project.

\section{References}

Baldwin, D.A., \& Moses, L.J. (1996). The ontogeny of social information gathering. Child Development, 67 (5), 19151939.

Baron-Cohen, S. (1991). Precursors to a theory of mind: understanding attention in others. In A. Whiten (Ed.), 
Natural theories of mind: Evolution, development and simulation of everyday mindreading (pp. 233-251). Oxford: Blackwell.

Bates, E., Camaioni, L., \& Volterra, V. (1975). The acquisition of performatives prior to speech. Merrill-Palmer Quarterly, 21, 205-224.

Blake, J. (2000). Routes to child language: Evolutionary and developmental precursors. Cambridge: Cambridge University Press.

Blake, J., O'Rourke, P., \& Borzellino, G. (1994). Form and function in the development of pointing and reaching gestures. Infant Behavior and Development, 17 (2), 195203.

Bretherton, I., McNew, S., \& Beeghly-Smith, M. (1981). Early person knowledge as expressed in gestural and verbal communication: when do infants acquire a 'theory of mind'? In M.E. Lamb \& L.R. Sherrod (Eds.), Infant social cognition: Empirical and theoretical considerations (pp. 333-373). Hillsdale, NJ: Erlbaum.

Butterworth, G. (1991). The ontogeny and phylogeny of joint visual attention. In A. Whiten (Ed.), Natural theories of mind: Evolution, development and simulation of everyday mindreading (pp. 223-232). Cambridge, MA: Blackwell.

Butterworth, G., Franco, F., McKennzie, B., Graupner, L., \& Todd, B. (2002). Dynamic aspects of visual event perception and the production of pointing by human infants. British Journal of Developmental Psychology, 20, 1-24.

Butterworth, G., \& Grover, L. (1990). Joint visual attention, manual pointing, and preverbal communication in human infancy. In M. Jeannerod (Ed.), Attention and performance XIII (pp. 605-624). Hillsdale, NJ: Erlbaum.

Call, J., \& Tomasello, M. (1996). The effect of humans on the cognitive development of apes. In A. Russon (Ed.), Reaching into thought: The minds of the great apes (pp. 371-403). Cambridge: Cambridge University Press.

Carpenter, M., Nagell, K., \& Tomasello, M. (1998). Social cognition, joint attention, and communicative competence from 9 to 15 months of age. Monographs of the Society for Research in Child Development, 63 (4), serial no. 255.

Franco, F., \& Butterworth, G. (1996). Pointing and social awareness: declaring and requesting in the second year. Journal of Child Language, 23 (2), 307-336.

Franco, F., \& Gagliano, A. (2001). Toddlers' pointing when joint attention is obstructed. First Language, 21 (63, Pt 3), 289-321.

Gusella, J.L., Muir, D., \& Tronick, E.A. (1988). The effect of manipulating maternal behavior during an interaction on three- and six-month-olds' affect and attention. Child Development, 59 (4), 1111-1124.

Henderson, L.M., Yoder, P.J., Yale, M.E., \& McDuffie, A. (2002). Getting the point: electrophysiological correlates of protodeclarative pointing. International Journal of Developmental Neuroscience, 20, 449-458.

Leung, E.H.L., \& Rheingold, H.L. (1981). Development of pointing as a social gesture. Developmental Psychology, 17 (2), 215-220.

Moore, C., \& Corkum, V. (1994). Social understanding at the end of the first year of life. Developmental Review, 14 (4), 349-372.

Moore, C., \& D'Entremont, B. (2001). Developmental changes in pointing as a function of attentional focus. Journal of Cognition and Development, 2 (2), 109-129.

Moses, L.J., Baldwin, D.A., Rosicky, J.G., \& Tidball, G. (2001). Evidence for referential understanding in the emotions domain at twelve and eighteen months. Child Development, 72 (3), 718-735.

Muir, D.W., \& Hains, S.M. (1993). Infant sensitivity to perturbations in adult facial, vocal, tactile and contingent stimulation during face-to-face interactions. In B. de Boysson-Bardies \& S. de Schonen (Eds.), Developmental neurocognition: Speech and face processing in the first year of life (Vol. 69, pp. 171185). Amsterdam: Elsevier.

Murphy, C.M., \& Messer, D.J. (1977). Mothers, infants and pointing: a study of a gesture. In H.R. Schaffer (Ed.), Studies in mother-infant interaction (pp. 325-354). New York: Academic.

Perucchini, P. (1997). Development of imperative and declarative functions of the pointing gesture. Giornale italiano di psicologia, 24 (4), 813-829.

Piaget, J. (1945/1962). Play, dreams, and imitation. New York: Norton.

Rochat, P., \& Striano, T. (1999). Social-cognitive development in the first year. In P. Rochat (Ed.), Early social cognition: Understanding others in the first months of life (pp. 3-34). Mahwah: Lawrence Erlbaum Associates.

Savage-Rumbaugh, E., \& Fields, W.M. (2000). Linguistic, cultural and cognitive capacities of bonobos (Pan paniscus). Culture and Psychology, 6 (2), 131-153.

Shwe, H.I., \& Markman, E.M. (1997). Young children's appreciation of the mental impact of their communicative signals. Developmental Psychology, 33 (4), 630-636.

Thiel, T. (1991). Interact (Version 6.5). Berlin, Germany.

Tomasello, M. (1999). The human adaptation for culture. Annual Review of Anthropology, 28, 509-529.

Tomasello, M., \& Camaioni, L. (1997). A comparison of the gestural communication of apes and human infants. Human Development, 40 (1), 7-24.

Tomasello, M., Farrar, M.J., \& Dines, J. (1984). Children's speech revisions for a familiar and an unfamiliar adult. Journal of Speech and Hearing Research, 27 (3), 359-363.

Tomasello, M., \& Haberl, K. (in press). Understanding attention: 12- and 18-month-olds know what's new for other persons. Developmental Psychology.

Werner, H., \& Kaplan, B. (1963). Symbol formation: An organismic-developmental approach to language and the expression of thought. New York: Wiley.

Received: 8 April 2003

Accepted: 20 August 2003 